\section{LUPUS SCIENCE\& MEDICINE}

To cite: Selvaraja M, Too CL, Tan LK, et al. Human leucocyte antigens profiling in Malay female patients with systemic lupus erythematosus: are we the same or different? Lupus Science \& Medicine 2022;9:e000554. doi:10.1136/ lupus-2021-000554

- Additional supplemental material is published online only. To view, please visit the journal online (http://dx.doi.org/10. 1136/lupus-2021-000554).

MS and CLT are joint first authors.

MA and SA-N are joint senior authors.

Received 14 August 2021 Accepted 8 January 2022

\section{Check for updates}

(c) Author(s) (or their employer(s)) 2022. Re-use permitted under CC BY-NC. No commercial re-use. See rights and permissions. Published by BMJ.

For numbered affiliations see end of article.

Correspondence to

Dr Chun Lai Too,

Immunogenetics Unit, Allergy and Immunology Research Centre,Block C7, Level 4, National Institutes of Health Complex, Ministry of Health Malaysia, Institute for Medical Research, Shah Alam, Selangor, Malaysia:; toocl@ moh.gov.my and Syafinaz Amin-Nordin, Department of Medical Microbiology, Faculty of Medicine and Health Sciences, Universiti Putra Malaysia,

Serdang, Selangor, Malaysia.; syafinaz@upm.edu.my

\title{
Human leucocyte antigens profiling in Malay female patients with systemic lupus erythematosus: are we the same or different?
}

\author{
Malarvili Selvaraja, ${ }^{1,2}$ Chun Lai Too (1) , ${ }^{3,4}$ Lay Kim Tan, ${ }^{3}$ Bee Tee Koay, ${ }^{5}$ \\ Maha Abdullah, ${ }^{2}$ Anim Md Shah, ${ }^{6}$ Masita Arip, ${ }^{7}$ Syafinaz Amin-Nordin ${ }^{2}$
}

\begin{abstract}
Objective SLE is a heterogeneous autoimmune disease, in terms of clinical presentation, incidence and severity across diverse ethnic populations. We investigated the human leucocyte antigens (HLA) profile (ie, HLA-A, HLA-B and HLA-C, HLA-DRB1, HLA-DQA1, HLA-DQB1, HLA-DPA1 and HLA-DPB1) in Malaysian Malay female patients with SLE and determined the generalisability of the published HLA risk factors across different ethnic populations globally including Malaysia.

Methods One hundred Malay female patients with SLE were recruited between January 2016 and October 2017 from a nephrology clinic. All patients were genotyped for HLA-A, HLA-B, HLA-C, HLA-DRB1, HLA-DQA1, HLA-DQB1, HLADPA1 and HLA-DPB1 alleles using PCR sequence-specific oligonucleotides method on Luminex platform. A total of 951 HLA genotyped population-based Malay control subjects was used for association testing by means of OR with $95 \%$ Cls. Results Our findings convincingly validated common associations between HLA-A*11 $\left(0 R=1.65, p=3.36 \times 10^{-3}\right.$, corrected $\left.\mathrm{P}(\mathrm{PC})=4.03 \times 10^{-2}\right)$ and $\mathrm{DQB1}{ }^{*} 05: 01(\mathrm{OR}=1.56$, $\mathrm{p}=2.02 \times 10^{-2}, \mathrm{PC}=$ non-significant) and SLE susceptibility in the Malay population. In contrast, $\mathrm{DQB} 1{ }^{*} 03: 01(\mathrm{OR}=0.51$, $\mathrm{p}=4.06 \times 10^{-4}, \mathrm{Pc}=6.50 \times 10^{-3}$ ) were associated with decreased risk of SLE in Malay population. Additionally, we also detected novel associations of susceptibility HLA genes (ie, HLA-B*38:02, DPA1*02:02, DPB1*14:01) and protective HLA genes (ie, DPA1*01:03). When comparing

\section{Key messages}

What is already known about this subject?

- Common SLE-related human leucocyte antigen (HLA) risk factors were highlighted in multiple ethnic populations.

- Nevertheless, studies have also shown populationspecific signals with the SLE-related HLA variants.

What does this study add?

- We validated the common and identified the novel SLE-associated HLA risk factors in Malay female patients with SLE.

- Analysis of current data and published data further support the notion that several HLA class II alleles (ie, DRB1*15, DQB1*03 and DQA1*01:02 alleles) are universally associated with risk of developing SLE in different ethnic populations and that these genetic effects are generalised to multiple ethnic populations of Caucasian, African and Asian descents.

How might this impact on clinical practice or future developments?

- Further genetic and pharmacogenetics research in SLE development is crucial for understanding the pathogenetic mechanism involved, which may pave a path for developing new and personalised therapeutic drugs for SLE.
\end{abstract} the current data with data from previously published studies from Caucasian, African and Asian populations, DRB1*15 alleles, DQB1*03:01 and DQA1*01:02 were corroborated as universal susceptibility and protective genes.

Conclusions This study reveals multiple HLA alleles associated with susceptibility and protection against risk of developing SLE in Malay female population with renal disorders. In addition, the published data from different ethnic populations together with our study further support the notion that the genetic effects from association with DRB1*15:01/02, DQB1*03:01 and DQA1*01:02 alleles are generalised to multiple ethnic populations of Caucasian, African and Asian descents.

\section{INTRODUCTION}

SLE is a complex, multisystem autoimmune disease characterised by a wide spectrum of clinical manifestations, and excessive immunological and laboratories abnormalities (ie, autoantibody production, complement activation and immune-complex deposition). SLE can lead to multiple organ damage, and is primarily affecting women with peak incidence occurs during childbearing age years. ${ }^{2}$ Despite advances in treatment, mortality among patients with SLE remains high with geographical variations. ${ }^{3}$

The prevalence of SLE ranges from 6.5 to 178.0 per 100000 person-years across different populations worldwide. ${ }^{4}$ Malaysia is a multiracial country with Malays as the largest ethnic group, followed by Chinese and 
Indians. The SLE prevalence in Malaysia has been documented as 43 per 100000 person-years with Malays having the second highest prevalence of SLE (ie, 33 per 100000 ) after Chinese (ie, 57 per 100000 ). ${ }^{5}$

Complete understanding of the pathophysiology of SLE remains elusive, but both genetics and environmental exposures are known to play a crucial role. ${ }^{6}$ A recent study demonstrated that the SLE heritability was estimated to be $44 \%$, with approximately $26 \%$ of phenotypic variance attributed to shared environmental factors. ${ }^{6}$ Nevertheless, the growing genetic heterogeneity in the pathogenesis of SLE makes it crucial for more investigation on patients with SLE of different sexes and varied ethnic populations around different parts of world. The knowledge gained will enable better understanding on the disease pathogenesis that could benefit pharmacogenetics and therapeutic strategies in SLE management.

Various genome-wide association studies have demonstrated the genetic and immunological differences attributed to SLE development. The human leucocyte antigen (HLA) class II, that is, DRB1 genes have been established as one of the prominent and strongest susceptibility genes for SLE development worldwide. ${ }^{78}$

Findings from several studies have demonstrated that HLA-DRB $1 * 03: 01$ and HLA-DRB1*15:01 alleles were robustly associated with increased risk of developing SLE in the Caucasian, African and East Asian populations. ${ }^{9-16}$ In contrast, HLA-DQB1*03:01 allele was associated with decreased risk of developing SLE in the Caucasian, East Asian and Southeast Asian populations. ${ }^{9} 1718$

In Malaysia, very few studies have investigated the HLA associations and risk of developing SLE with a limited number of HLA loci, for example, mainly on HLA class II genes. ${ }^{19-21}$ Although these studies focused on investigation of HLA associations in the major ethnic group from Malaysia, namely the Malays, no consistent trend of associations was observed between different HLA alleles and SLE risk. Furthermore, none of the identified HLA risk factors were overlapped among these three studies. The plausible explanations could be due to the limitations with different genotyping platforms used, relatively small sample size as well as the underlying clinical phenotypic heterogeneity of SLE. Our research group has recently reported the association between HLA-DRB1 alleles and risk of developing SLE in the Malay population. ${ }^{22}$ We demonstrated that HLA-DRB1*04:01, DRB1*04:05, DRB1*15:02 and DRB1*16:02 alleles were susceptible to increased risk of SLE, while DRB1*12:01 and DRB1*12:02 alleles were associated with decreased risk for SLE development in this study. Thus, we aimed to overcome some of the previous studies limitations by profiling a broader array of HLA polymorphisms to include the classical HLA class I loci (ie, HLA-A, HLA-B and HLA-C) and extended HLA class II loci (ie, HLA-DRB1, HLA-DQA1, HLA-DQB1, HLA-DPA1 and HLA-DPB1) in a larger, well-characterised Malaysian Malay female patients with SLE with renal manifestations. We compared the HLA allelic frequency distributions and associations for risk of developing SLE using the HLA genotypes data obtained from the largest population-based normal controls with matched Malay ethnicity. ${ }^{23}$ We also compared our data with the published significant studies to address the question how much of the identified HLA risk factors in multiple ethnic populations can be translated across the Malaysian Malay population and finally elucidate the generalisability of HLA risk factors in SLE populations of Caucasian, African and Asian descents.

\section{METHODS}

\section{Study population}

This is a single-centre study comprising 100 Malaysian Malay female patients with SLE. The patients with SLE were aged between 16 and 53 years and were recruited from the Nephrology Clinic, Hospital Serdang Malaysia between January 2016 and October 2017. The study design and demographic characteristics for the patients with SLE have been described elsewhere.$^{22}$ Briefly, all the SLE cases were diagnosed according to the 1997 American College of Rheumatology (ACR) criteria. ${ }^{24}$

\section{Genomic DNA extraction}

Ten millilitres of peripheral blood samples were collected from all recruited participants in EDTA tubes. The genomic DNA was extracted from the whole blood samples using the QIAamp DNA Blood Mini Extraction Kit (Qiagen, Hilden, Germany, cat no: 51104) according to the manufacturer's instructions. DNA purity and concentration were estimated using the Nanodrop 1000 spectrophotometer (Thermo Scientific, USA). All DNA samples were stored at $-20^{\circ} \mathrm{C}$ until further use.

\section{Classical HLA class I and class II genotyping}

All patients with SLE were genotyped for classical HLA class I (ie, HLA-A, HLA-B and HLA-C) and HLA class II alleles (ie, HLA-DRB1, HLA-DQA1, HLA-DQB1, HLADPA1 and HLA-DPB1) using PCR sequence-specific oligonucleotides (PCR-SSO) probe hybridisation method (LIFECODES HLA SSO Typing Kit, Gene Probe, USA) with Luminex Multi-Analyte Profiling System (xMAP, Luminex, Texas, USA), according to manufacturer's instructions. The assignment of the specific HLA alleles was accomplished using the Quick Type for Life Match 2.6.1 software for Gen-Probe analysis.

\section{Statistical analysis}

The HLA allele frequencies for Malay patients with SLE and ethnically matched normal controls were obtained by direct counting. An individual was considered homozygous if only one allele was detected in the genotyping assay, and the allele frequency was counted twice.

The complete HLA genotypes dataset for a total of 951 Peninsular Malaysian Malay normal individuals, comprising five loci of classical HLA alleles namely HLA-A, HLA-B, HLA-C, HLA-DRB1 and HLA-DQB1 were obtained from the multicenter Malaysian Epidemiological Investigation of Rheumatoid Arthritis (MyEIRA) 
population-based case-control study. ${ }^{23}$ The details of this study population have been described elsewhere. ${ }^{23}{ }^{25}$ The frequencies and ORs with 95\% CIs of the HLA alleles were compared between the patients with SLE and normal control subjects using the $\chi^{2}$ or Fisher's exact test, where appropriate. Adjustment for multiple comparisons were performed using the Bonferroni correction method. The corrected $\mathrm{P}(\mathrm{Pc})$ values were calculated by multiplying the observed $\mathrm{p}$ values by the number of alleles tested: for 2-digit HLA alleles, 12 for HLA-A, 21 for HLA-B, 10 for HLA-C, 13 for HLA-DRB1, 6 for HLA-DQA1, 5 for HLA-DQB1, 3 for HLA-DPA1 and 13 for HLA-DPB1. The number of alleles tested for 4-digit HLA was 22 for HLA-A, 46 for HLA-B, 21 for HLA-C, 26 for HLA-DRB1, 11 for HLA-DQA1, 16 for HLA-DQB1, 5 for HLA-DPA1 and 15 for HLA-DPB1. A probability of 0.05 (two-tailed) was used as a significance threshold.

For the normal controls dataset, we did not perform the experimental classical HLA-DQA1, HLA-DPA1 and HLA-DPB1 genotyping. Nevertheless, the MyEIRA study subjects were genotyped using the Illumina iSelect HD custom genotyping array designed by the Immunochip Consortium (Immunochip Illumina, San Diego, California, USA). The details of the classical HLA imputation for the dataset were previously described.$^{26}$ Briefly, a total of 6125 single nucleotide polymorphisms (SNPs) between position 29 and $34 \mathrm{Mb}$ in the HLA region on chromosome 6 (GRCh37) were extracted from the postquality control Immunochip dataset. We imputed the classical two-digit and four-digit HLA alleles by using the extracted SNP genotypes from the HLA region, along with SNPs from the Pan Asian reference panel. ${ }^{27}$ In this Malay normal control dataset, we imputed 6 two-digit HLADQA1, 3 HLA-DPA1 and 14 HLA-DPB1 alleles. For the four-digit HLA imputation, we obtained 8 HLA-DQA1, 4 HLA-DPA1 and 17 HLA-DPB1 alleles. We performed the association analysis by comparing the allelic frequencies between experimental genotyped patients with SLE and imputed-genotyped normal controls for HLA-DQA1, HLA-DPA1 and HLA-DPB1 alleles.

The two HLA loci (ie, HLA-DRB1 DQB1), three HLA loci (ie, HLA-A DRB1 DQB1) and five HLA loci (ie, HLA-A B C $\sim \mathrm{DRB} 1 \sim \mathrm{DQB} 1$ ) haplotype frequencies were estimated using Expectation Maximisation (EM) algorithm included in the ARLEQUIN software (Arlequin V.3.5.2.2 software).$^{28}$ We compared the obtained haplotype frequencies and performed the association analysis between the patients with SLE and the ethnically matched normal controls for risk of developing SLE. Standard software (SPSS V.17.0 for Windows, SPSS, Chicago, Illinois, USA) was used for statistical analyses.

\section{Comparative analysis with published data}

To determine the generalisability or universal HLA risk factors for SLE development across different ethnic populations worldwide, we compared the current findings with the published significant SLE-associated HLA alleles from different populations/ethnic groups (ie,

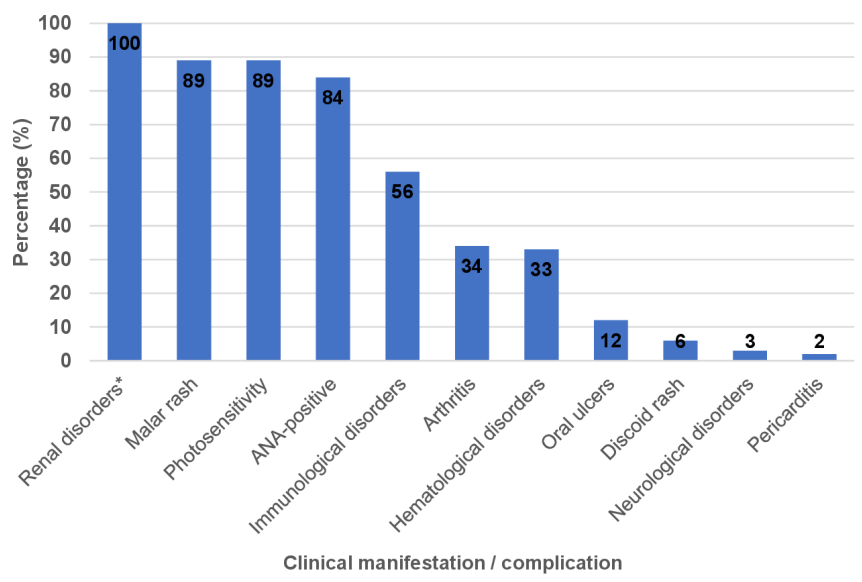

Figure 1 The percentage of various clinical manifestations and/or complications among the Malay female patients with SLE $(n=100)$. *All the patients with SLE have mild to severe renal disorders (ie, presence of proteinuria $>0.5 \mathrm{~g} / \mathrm{day}$; or active urinary sediments with casts and/or $>5$ red cells per high-power field and/or biopsy-proven lupus nephritis).

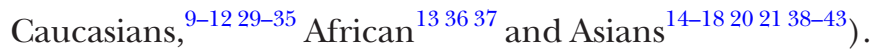
We restricted our selection of published SLE-associated HLA risk factors which were genotyped by molecular methods.

\section{Patient and public involvement}

There is no involvement of patient and public in the (i) setting/shaping of research questions, (ii) planning research, (iii) conducting research, (iv) interpreting findings and (v) sharing and/or using the research knowledge in this study.

\section{RESULTS \\ Characteristics and clinical manifestations among the patients with SLE}

All the recruited patients with SLE were female with mean age of SLE onset (years \pm SEM) of $30.87 \pm 0.78$ years. The mean disease duration was 82 months. The main clinical manifestations based on the 1997 ACR classification for SLE, are shown in figure 1 . All the patients with SLE have mild to severe renal disorders (ie, presence of proteinuria $>0.5 \mathrm{~g} /$ day; or active urinary sediments with casts and/or $>5$ red cells per high-power field and/or biopsyproven lupus nephritis). Other common clinical manifestations observed in this study was malar rash (89\%) and photosensitivity (89\%). ANA and double-stranded DNA antibodies were detected in $83.8 \%$ and $56.6 \%$ among the studied patients with SLE.

\section{HLA-A, HLA-B and HLA-C alleles and the risk of developing SLE}

A total of 12 HLA-A, 21 HLA-B and 10 HLA-C 2-digit allelic groups were detected in the patients with SLE (online supplemental table S1). The number of detected 4-digit classical HLA subtype alleles increased two times for the studied HLA loci, for example, 22 HLA-A, 46 HLA-B and 21 HLA-C alleles (online supplemental table 
Table 1 OR and $95 \% \mathrm{Cl}$ of the HLA-A and HLA-B alleles significantly associated with risk of developing SLE in Malaysian female population

\begin{tabular}{|c|c|c|c|c|c|c|c|}
\hline & & $\begin{array}{l}\text { Patients with } \\
\text { SLE }(2 n=200)\end{array}$ & $\begin{array}{l}\text { Normal controls } \\
(2 n=1902)\end{array}$ & & & & \\
\hline HLA gene & HLA allele & $\mathrm{AC}(\mathrm{AF})$ & $A C(A F)$ & OR & $95 \% \mathrm{Cl}$ & $P$ value & Pc value \\
\hline \multicolumn{8}{|c|}{ Classical two-digit HLA allele } \\
\hline HLA-A & HLA-A*11 & $52(0.260)$ & $334(0.176)$ & 1.65 & 1.18 to 2.31 & $3.36 \times 10^{-3}$ & $4.03 \times 10^{-2}$ \\
\hline HLA-B & HLA-B*38 & $18(0.090)$ & $72(0.038)$ & 2.51 & 1.47 to 4.31 & $7.94 \times 10^{-4}$ & $1.67 \times 10^{-2}$ \\
\hline \multicolumn{8}{|c|}{ Classical four-digit HLA allele } \\
\hline HLA-A & HLA-A*11:01 & $48(0.240)$ & $326(0.171)$ & 1.53 & 1.08 to 2.16 & $2.59 \times 10^{-2}$ & ns \\
\hline HLA-A & HLA-A*33:01 & $29(0.145)$ & $160(0.084)$ & 1.85 & 1.21 to 2.83 & $4.19 \times 10^{-3}$ & ns \\
\hline HLA-A & HLA-A*33:03 & $1(0.005)$ & $79(0.042)$ & 0.12 & 0.02 to 0.84 & $1.02 \times 10^{-2}$ & ns \\
\hline HLA-B & HLA-B*38:02 & $18(0.090)$ & $72(0.038)$ & 2.51 & 1.47 to 4.31 & $5.29 \times 10^{-4}$ & ns \\
\hline
\end{tabular}

$\mathrm{AC}$, allele count; AF, allele frequency; HLA, human leucocyte antigen; $2 n$, allelic count; ns, non-significant; Pc, corrected $p$ value.

S2). Our data demonstrated that HLA-A*11 allelic group was associated with increased risk for developing SLE in the Malay female population $(\mathrm{OR}=1.65,95 \% \mathrm{CI}=1.18$ to 2.31). Notably, we observed significant novel association between HLA-B $* 38$ and risk of SLE $(\mathrm{OR}=2.51,95 \%$ $\mathrm{CI}=1.47$ to 4.31 ) (table 1 ). No significant association was observed between HLA-C allelic groups and risk of SLE in the Malay population (data not shown).

Analysis by four-digit classical HLA class I allele subtypes further revealed that HLA-A*11:01 and HLAB*38:02 subtype alleles were significantly associated with increased risk of SLE in the Malay population $(\mathrm{OR}=1.53$; $95 \% \mathrm{CI}=1.08$ to 2.16$)$ and $(\mathrm{OR}=2.51,95 \% \mathrm{CI}=1.47$ to 4.31) (table 1). Considering sex differences may be a concern for risk association analysis, we further stratified the normal individuals by sex and conducted the analysis using the female-only controls $(n=814)$. Our data revealed concordance findings between overall normal controls versus the female-only controls (online supplemental tables S1 and S2).

\section{HLA-DQA1, HLA-DQB1, HLA-DPA1 and HLA-DPB1 alleles and the risk of developing SLE}

In this study, we identified 6 HLA-DQA1, 5 HLA-DQB1, 3 HLA-DPA1 and 13 HLA-DPB1 2-digit allelic groups in the dataset of patients with SLE. Similar to that of identified HLA class I subtype alleles, the number of identified 4-digit HLA class II alleles in the patients with SLE were 11, 16, 5 and 15 for HLA-DQA1, HLA-DQB1, HLA-DPA1 and HLA-DPB1 alleles, respectively (online supplemental tables S1 and S2). In addition to the previously reported HLA-DRB1 alleles associated with risk of SLE, ${ }^{22}$ our data showed that HLA-DQB $1 * 05 \quad(\mathrm{OR}=1.84,95 \% \mathrm{CI}=1.37$ to 2.48) were significantly associated with increased risk for developing SLE among the Malay female population. In contrast, we observed significant decreased risk between patients with SLE HLA-DQB $1 * 03$ allele $(\mathrm{OR}=0.50,95 \%$ $\mathrm{CI}=0.36$ to 0.71 ) when compared with the ethnically matched normal controls (table 2).
Next, we compared the extended classical HLA class II alleles, that is, HLA-DQA1, HLA-DPA1 and HLA-DPB1 alleles between patients with SLE and normal controls, our data demonstrated several susceptibility alleles associated with risk of SLE development, for example, HLA-DQA $1 * 01 \quad(\mathrm{OR}=2.01), \quad$ HLA-DPA $1 * 02 \quad(\mathrm{OR}=1.88)$, HLA-DPB $1 * 05(\mathrm{OR}=1.66)$ and HLA-DPB $1 * 14(\mathrm{OR}=2.91)$ (table 2). Our findings also revealed that HLA-DQA $1 * 06$ $(\mathrm{OR}=0.33), \mathrm{HLA}-\mathrm{DPA} 1 * 01(\mathrm{OR}=0.55)$ and HLA-DPB $1 * 04$ $(\mathrm{OR}=0.43)$ were associated with decreased risk of developing SLE. All the observed two-digit HLA associations were further confirmed with four-digit resolution alleles of the studied loci (table 2). It is noteworthy that majority of the observed associations remained statistically significant after Bonferroni correction for multiple comparisons (table 2).

Taking into consideration that genetic variations were detected in the normal controls used for HLA association analysis in this study, as measured by the Hardy-Weinberg equilibrium, ${ }^{23}$ we further confirmed our findings using two published Malay control groups with available HLA genotypes. ${ }^{44}$ The observed trend of associations and risk effects for the HLA class I and class II alleles were corroborated with our initial findings, although some of these associations were statistically non-significant (online supplemental table S3).

\section{Associations between HLA haplotypes and risk of developing SLE}

We constructed two-loci (ie, HLA-DRB1 DQB1), threeloci (ie, HLA-A DRB1 DQB1) and five loci (ie, HLA$\mathrm{A} \sim \mathrm{B} \sim \mathrm{C} \sim \mathrm{DRB} 1 \sim \mathrm{DQB} 1)$ haplotype association analysis between the patients with SLE and normal controls. We identified 79, 139 and 177 haplotypes for two-loci, threeloci and five-loci HLA alleles in the SLE patients' group. In two-loci haplotype analysis, we observed that 10 haplotypes were significantly associated with risk of developing SLE in the Malay population with OR ranging from 3.07 to 50.0. On the other hand, only one two-locus haplotype, 
Table 2 ORs and 95\% Cls of the HLA-DQA1, HLA-DPA1 and HLA-DPB1 alleles significantly associated with risk of developing SLE in Malaysian female population

\begin{tabular}{|c|c|c|c|c|c|c|c|}
\hline & & $\begin{array}{l}\text { Patients with } \\
\text { SLE }(2 n=200)\end{array}$ & $\begin{array}{l}\text { Normal } \\
\text { controls } \\
(2 n=1902) \\
\end{array}$ & & & & \\
\hline HLA gene & HLA allele & $A C(A F)$ & $A C(A F)$ & OR & $95 \% \mathbf{C l}$ & $P$ value & Pc value \\
\hline \multicolumn{8}{|c|}{ Classical two-digit HLA allele } \\
\hline \multirow[t]{2}{*}{$H L A-D Q A 1$} & $H L A-D Q A 1^{*} 01$ & $124(0.620)$ & $827(0.448)$ & 2.01 & 1.49 to 2.71 & $3.85 \times 10^{-6}$ & $2.31 \times 10^{-5}$ \\
\hline & $H L A-D Q A 1^{*} 06$ & $22(0.110)$ & $504(0.273)$ & 0.33 & 0.21 to 0.52 & $5.22 \times 10^{-7}$ & $3.13 \times 10^{-6}$ \\
\hline \multirow[t]{2}{*}{$H L A-D Q B 1$} & $H L A-D Q B 1{ }^{*} 03$ & $46(0.230)$ & 707 (0.372) & 0.51 & 0.35 to 0.74 & $7.00 \times 10^{-5}$ & $3.50 \times 10^{-4}$ \\
\hline & $H L A-D Q B 1^{*} 05$ & $92(0.460)$ & $601(0.316)$ & 1.84 & 1.37 to 2.48 & $3.78 \times 10^{-5}$ & $1.89 \times 10^{-4}$ \\
\hline \multirow[t]{2}{*}{$H L A-D P A 1$} & $H L A-D P A 1^{*} 01$ & $57(0.285)$ & $774(0.420)$ & 0.55 & 0.40 to 0.76 & $2.29 \times 10^{-4}$ & $6.87 \times 10^{-4}$ \\
\hline & HLA-DPA1*02 & $128(0.640)$ & $897(0.486)$ & 1.88 & 1.39 to 2.54 & $3.70 \times 10^{-5}$ & $1.11 \times 10^{-4}$ \\
\hline \multirow[t]{3}{*}{$H L A-D P B 1$} & $H L A-D P B 1{ }^{*} 04$ & $24(0.120)$ & $442(0.240)$ & 0.43 & 0.28 to 0.67 & $1.27 \times 10^{-4}$ & $1.65 \times 10^{-3}$ \\
\hline & $H L A-D P B 1{ }^{*} 05$ & $53(0.265)$ & $329(0.178)$ & 1.66 & 1.19 to 2.32 & $2.85 \times 10^{-3}$ & $3.71 \times 10^{-2}$ \\
\hline & $H L A-D P B 1 * 14$ & $13(0.065)$ & $43(0.023)$ & 2.91 & 1.54 to 5.51 & $6.05 \times 10^{-4}$ & $7.87 \times 10^{-3}$ \\
\hline \multicolumn{8}{|c|}{ Classical four-digit HLA allele } \\
\hline \multirow[t]{3}{*}{$H L A-D Q A 1$} & $H L A-D Q A 1 * 01: 02$ & $66(0.330)$ & $387(0.210)$ & 1.85 & 1.35 to 2.54 & $1.03 \times 10^{-4}$ & $1.13 \times 10^{-3}$ \\
\hline & $H L A-D Q A 1^{*} 03: 01$ & $3(0.015)$ & $184(0.099)$ & 0.14 & 0.04 to 0.43 & $7.83 \times 10^{-5}$ & $8.61 \times 10^{-4}$ \\
\hline & $H L A-D Q A 1 * 06: 01$ & $22(0.110)$ & $504(0.273)$ & 0.33 & 0.21 to 0.52 & $5.22 \times 10^{-7}$ & $5.74 \times 10^{-6}$ \\
\hline \multirow[t]{3}{*}{$H L A-D Q B 1$} & $H L A-D Q B 1 * 03: 01$ & $35(0.175)$ & $562(0.296)$ & 0.50 & 0.36 to 0.71 & $4.06 \times 10^{-4}$ & $6.50 \times 10^{-3}$ \\
\hline & $H L A-D Q B 1 * 05: 01$ & $38(0.190)$ & $248(0.130)$ & 1.56 & 1.07 to 2.28 & $2.02 \times 10^{-2}$ & ns \\
\hline & $H L A-D Q B 1 * 05: 02$ & $50(0.25)$ & $270(0.142)$ & 2.02 & 1.42 to 2.85 & $5.21 \times 10^{-5}$ & $8.34 \times 10^{-4}$ \\
\hline \multirow[t]{2}{*}{$H L A-D P A 1$} & $H L A-D P A 1 * 01: 03$ & $56(0.280)$ & $774(0.419)$ & 0.54 & 0.39 to 0.74 & $1.32 \times 10^{-4}$ & $6.61 \times 10^{-4}$ \\
\hline & HLA-DPA1*02:02 & $88(0.440)$ & $570(0.309)$ & 1.76 & 1.31 to 2.36 & $1.68 \times 10^{-4}$ & $8.40 \times 10^{-4}$ \\
\hline \multirow[t]{6}{*}{$H L A-D P B 1$} & $H L A-D P B 1 * 01: 01$ & $19(0.050)$ & $47(0.025)$ & 2.01 & 1.00 to 4.05 & $4.55 \times 10^{-2}$ & ns \\
\hline & $H L A-D P B 1^{*} 02: 02$ & $16(0.080)$ & $74(0.040)$ & 2.08 & 1.87 to 3.65 & $9.07 \times 10^{-3}$ & ns \\
\hline & $H L A-D P B 1 * 04: 01$ & $21(0.105)$ & $343(0.186)$ & 0.51 & 0.32 to 0.82 & $4.45 \times 10^{-3}$ & ns \\
\hline & $H L A-D P B 1^{*} 04: 02$ & $3(0.015)$ & $99(0.054)$ & 0.27 & 0.08 to 0.85 & $1.70 \times 10^{-2}$ & ns \\
\hline & $H L A-D P B 1^{*} 05: 01$ & $53(0.265)$ & $329(0.178)$ & 1.66 & 1.19 to 2.32 & $2.85 \times 10^{-3}$ & ns \\
\hline & $H L A-D P B 1^{*} 14: 01$ & $13(0.065)$ & $43(0.023)$ & 2.91 & 1.54 to 5.51 & $6.05 \times 10^{-4}$ & $1.09 \times 10^{-2}$ \\
\hline
\end{tabular}

$\mathrm{AC}$, allele count; AF, allele frequency; HLA, human leucocyte antigen; $2 n$, allelic count; ns, non-significant; Pc, corrected $p$ value.

that is, DRB1*12:02 DQB1*03:01, was negatively associated with risk of SLE (OR=0.13) (table 3). Further analysis with three-locus haplotype revealed that only four haplotypes were positively associated with risk of developing SLE with ORs between 2.77 and 29.38 (table 3). Only 3 out of 177 five-locus haplotypes (1.7\%) were found to be associated with susceptibility to SLE development in the Malaysian female patients with SLE (table 3).

DRB1 and DQB1 alleles are in linkage disequilibrium. In order to elucidate which DRB1 and DQB1 alleles were responsible for the observed risk associations, conditional logistic regression analysis between them in SLE was performed (table 4). The protective association of DRB1*12:01 allele remained significant, when conditioned on DRB $1 * 04: 05$, DRB $1 * 12: 02$, DRB1*15:02, DQB1*03:01, DQB1*05:01 or DQB1*05:02. The significant susceptibility association of DQB $1 * 05: 02$ allele was observed, when conditioned on DRB1*04:05,
DRB1*12:01, DRB1*12:02, DRB1*15:02, DQB1*03:01 or DQB1*05:01. Thus, significant association for DRB1*12:01 and DQB1*05:02 were observed, when conditioned on the associated DRB1 and DQB1 alleles, indicating an independent role for these alleles in SLE.

Comparative analysis for the SLE-associated HLA alleles and risk of SLE across different populations

We compared the published SLE-associated HLA susceptibility and protective alleles across different populations/ethnic groups to address the question as to how far the identified SLE risk factors in Caucasians, Africans and Asians can be translated across different ethnic populations including the Malaysian Malays. The data are presented in table 5. The HLA-DRB $1 * 03$ and HLADRB $1 * 15$ alleles were the most common HLA susceptibility alleles significantly associated with increased risk of SLE in both Caucasian and Asian populations. However, 
Table 3 ORs and 95\% Cls of the HLA two-loci, three-loci and five-loci haplotypes significantly associated with risk of developing SLE in Malaysian female population

\begin{tabular}{|c|c|c|c|c|}
\hline HLA haplotype & $\begin{array}{l}\text { Patients with } \\
\text { SLE } \\
(2 n=200), \text { HF }\end{array}$ & $\begin{array}{l}\text { Normal controls } \\
(2 n=1902), \text { HF }\end{array}$ & OR & $95 \% \mathbf{C l}$ \\
\hline \multicolumn{5}{|l|}{ HLA-DRB1 DQB1 } \\
\hline$D R B 1 * 15: 02 \sim D Q B 1{ }^{*} 03: 01$ & $10(0.050)$ & $2(0.001)$ & 50.00 & 10.88 to 229.88 \\
\hline$D R B 1 * 04: 05 \sim D Q B 1{ }^{*} 03: 01$ & $7(0.033)$ & $4(0.002)$ & 17.21 & 4.99 to 59.32 \\
\hline$D R B 1 * 12: 02 \sim D Q B 1{ }^{*} 05: 02$ & $6(0.031)$ & $19(0.010)$ & 3.07 & 1.21 to 7.77 \\
\hline$D R B 1 * 12: 02 \sim D Q B 1{ }^{*} 03: 01$ & $4(0.021)$ & $267(0.140)$ & 0.13 & 0.05 to 0.34 \\
\hline$D R B 1 * 15: 01 \sim D Q B 1{ }^{*} 03: 01$ & $4(0.020)$ & $5(0.003)$ & 7.74 & 2.06 to 29.07 \\
\hline DRB1*04:05 DQB1 ${ }^{*} 05: 02$ & $3(0.015)$ & $1(0.001)$ & 28.95 & 3.00 to 279.64 \\
\hline$D R B 1 * 12: 02 \sim D Q B 1 * 05: 01$ & $3(0.015)$ & $2(0.001)$ & 14.47 & 2.40 to 87.10 \\
\hline DRB1*04:05 DQB1*02:02 & $3(0.015)$ & $2(0.001)$ & 14.47 & 2.40 to 87.10 \\
\hline$D R B 1 * 07: 01 \sim D Q B 1 * 05: 02$ & $3(0.015)$ & $2(0.001)$ & 14.47 & 2.40 to 87.10 \\
\hline DRB1*15:02 DQB1*02:01 & $2(0.100)$ & $1(0.001)$ & 19.20 & 1.73 to 212.72 \\
\hline$D R B 1 * 16: 02 \sim D Q B 1{ }^{*} 03: 01$ & $2(0.009)$ & $2(0.001)$ & 9.60 & 1.34 to 68.50 \\
\hline \multicolumn{5}{|l|}{$H L A-A \sim D R B 1 \sim D Q B 1$} \\
\hline$A^{*} 11: 01 \sim D R B 1^{*} 15: 02 \sim D Q B 1 * 05: 01$ & $10(0.05)$ & $31(0.02)$ & 3.18 & 1.53 to 6.58 \\
\hline$A^{*} 24: 02 \sim D R B 1{ }^{*} 15: 02 \sim D Q B 1 * 03: 01$ & $6(0.03)$ & $2(0.001)$ & 29.38 & 5.89 to 146.57 \\
\hline$A^{*} 34: 01 \sim D R B 1^{*} 15: 02 \sim D Q B 1 * 05: 02$ & $6(0.03)$ & $21(0.01)$ & 2.77 & 1.10 to 6.95 \\
\hline$A^{*} 11: 01 \sim D R B 1 * 15: 02 \sim D Q B 1 * 05: 02$ & $6(0.03)$ & $16(0.009)$ & 3.65 & 1.41 to 9.43 \\
\hline \multicolumn{5}{|l|}{$H L A-A \sim B \sim C \sim D R B 1 \sim D Q B 1$} \\
\hline$A^{*} 33: 01 \sim B^{*} 44: 03 \sim C^{*} 07: 01 \sim D R B 1^{*} 07: 01 \sim D Q B 1^{*} 02: 02$ & $6(0.030)$ & $21(0.011)$ & 2.77 & 1.10 to 6.95 \\
\hline$A^{*} 11: 01 \sim B^{\star} 35: 05 \sim C^{*} 04: 01 \sim D R B 1{ }^{*} 15: 02 \sim D Q B 1{ }^{*} 05: 01$ & $4(0.020)$ & $1(0.0005)$ & 38.8 & 4.31 to 348.83 \\
\hline$A^{*} 33: 01 \sim B^{\star} 58: 01 \sim C^{*} 03: 02 \sim D R B 1{ }^{*} 03: 01 \sim D Q B 1{ }^{*} 02: 01$ & $4(0.020)$ & $7(0.004)$ & 5.52 & 1.60 to 19.04 \\
\hline
\end{tabular}

HF, haplotype frequency; HLA, human leucocyte antigen; 2n, haplotype count.

only the HLA-DRB $1 * 15$ association was validated in our study while the HLA-DRB $1 * 03$ alleles frequency was comparable between Malay patients with SLE (7\%) and the ethnically matched controls (5\%). Interestingly, DRB $1 * 15$ risk alleles seemed to be shared, but the risk signal observed appeared to be population specific, that is, HLA-DRB1*15:01 in Caucasians, Saudis, Japanese, Koreans and Taiwanese and HLA-DRB1*15:02 in Southeast Asians, that is, Thais and Malaysian populations. On the other hand, HLA-DQB1*03:01 allele was convincingly validated as protective allele in our study and across the studied populations included in this study. Interestingly, we observed several HLA alleles, for example, HLAB*38:02, and DRB1*16:02 as susceptibility genes, which were not reported in any of the published data from these studied populations (table 5).

\section{DISCUSSION}

This study is the first comprehensive classical HLA genotyping analysis to identify susceptibility and protective variants in Malaysian female patients with SLE for the extensive association signals previously observed across the HLA region globally. In this study, our data revealed that multiple HLA alleles were associated with SLE susceptibility and protection in the Malaysian female population. Notably, we observed strong significant association between the HLA-B*38:02 $(\mathrm{OR}=2.51)$ allele and SLE in Malay population as novel result. In addition, our observations on positive associations were extended to several HLA class II alleles, for example, DQA1*01:02, DPA $1 * 02: 02$ and negative associations to the DQA $1 * 03: 01$ and DPA1*01:03 alleles in the Malay SLE population. When comparing the current findings with the previously published HLA association data from Caucasian, African, Asian and Southeast Asian populations, DRB1*15 and DQB $1 * 03$ alleles were corroborated as universal susceptibility and protective genes in different ethnic populations worldwide.

In our HLA class I genotyping analysis, we confirmed the previous association of $\mathrm{A}^{*} 11$ in the Malay patients with SLE, ${ }^{21}$ and further demonstrated the risk variant signal as $A^{*} 11: 01$ allele. Noticeably, we identified that the $B^{*} 38: 02$ allele was significantly associated with risk of SLE $(\mathrm{OR}=2.51)$, which has not been reported in previous studies. In Caucasians, several reports have consistently demonstrated that the HLA-B*08/*08:01 was significantly increased in patients with SLE. ${ }^{31} 3235$ It is noteworthy that HLA-B*08 is one of the alleles residing within 


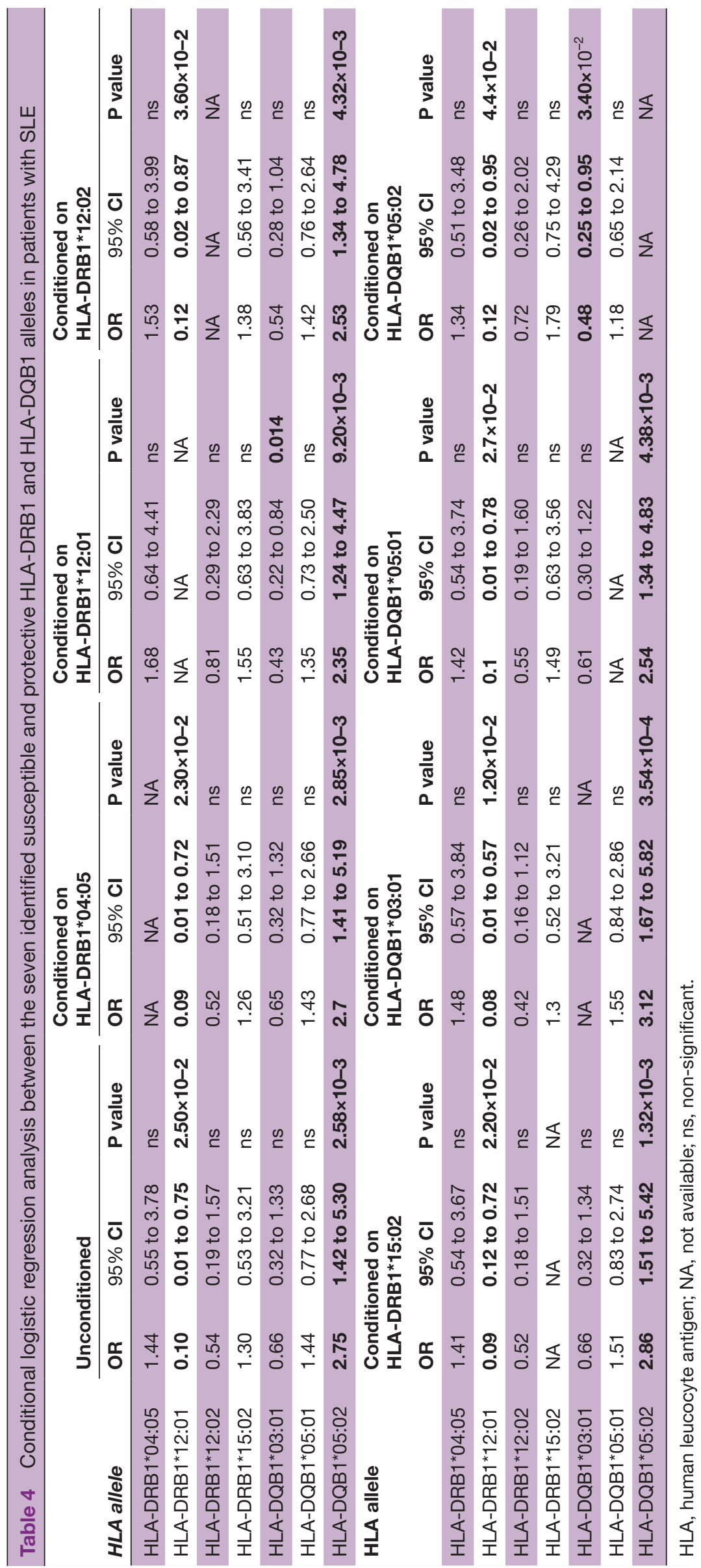




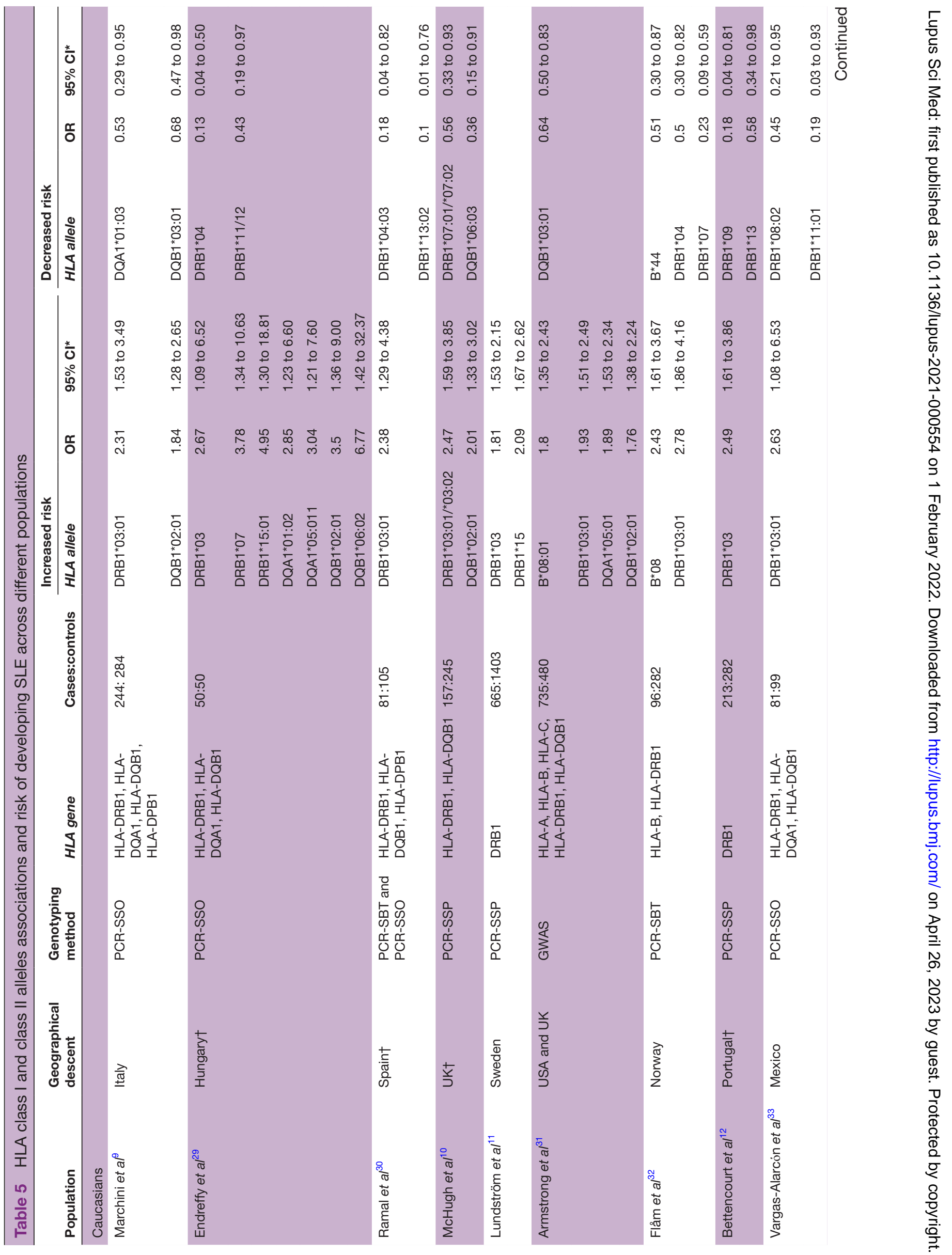




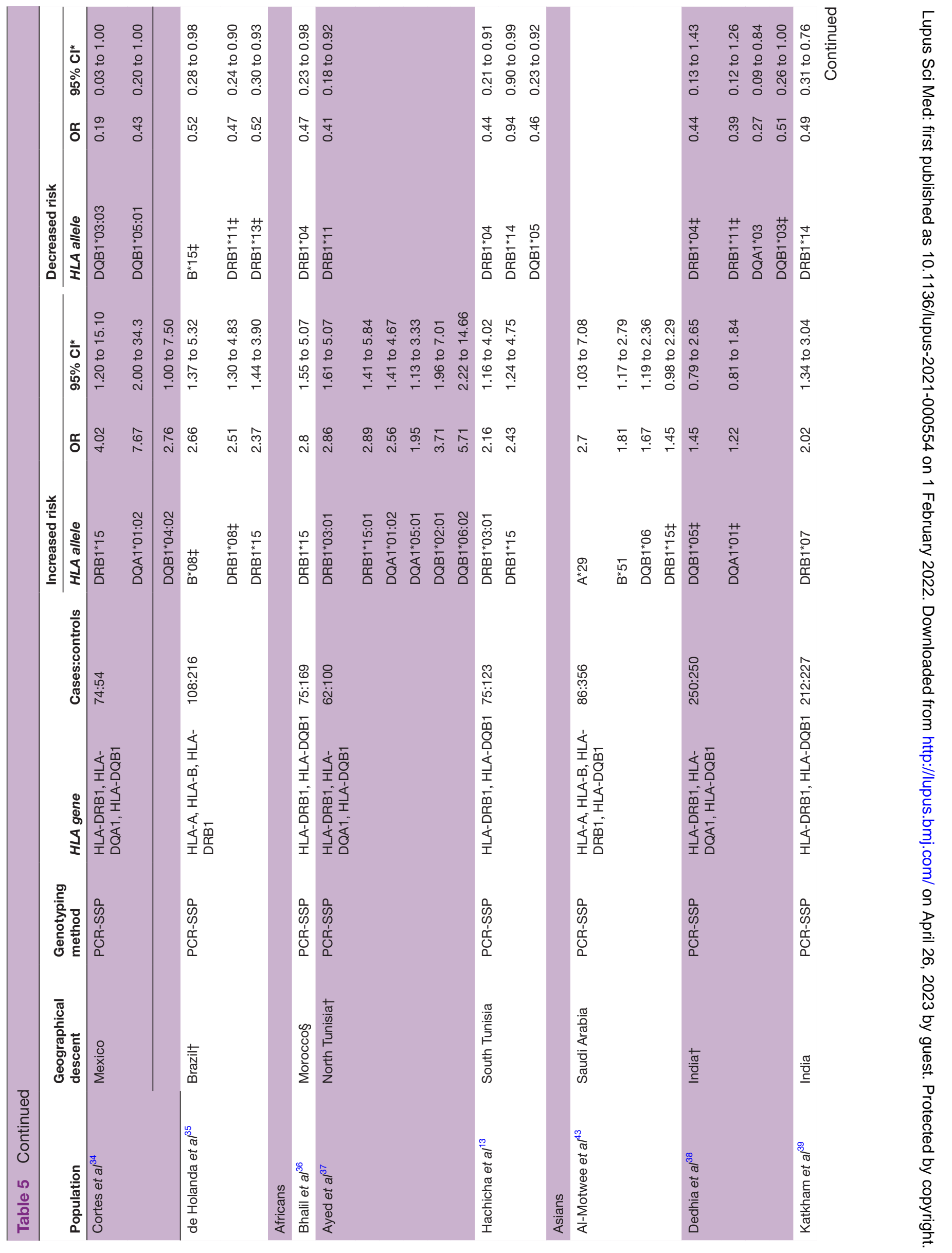




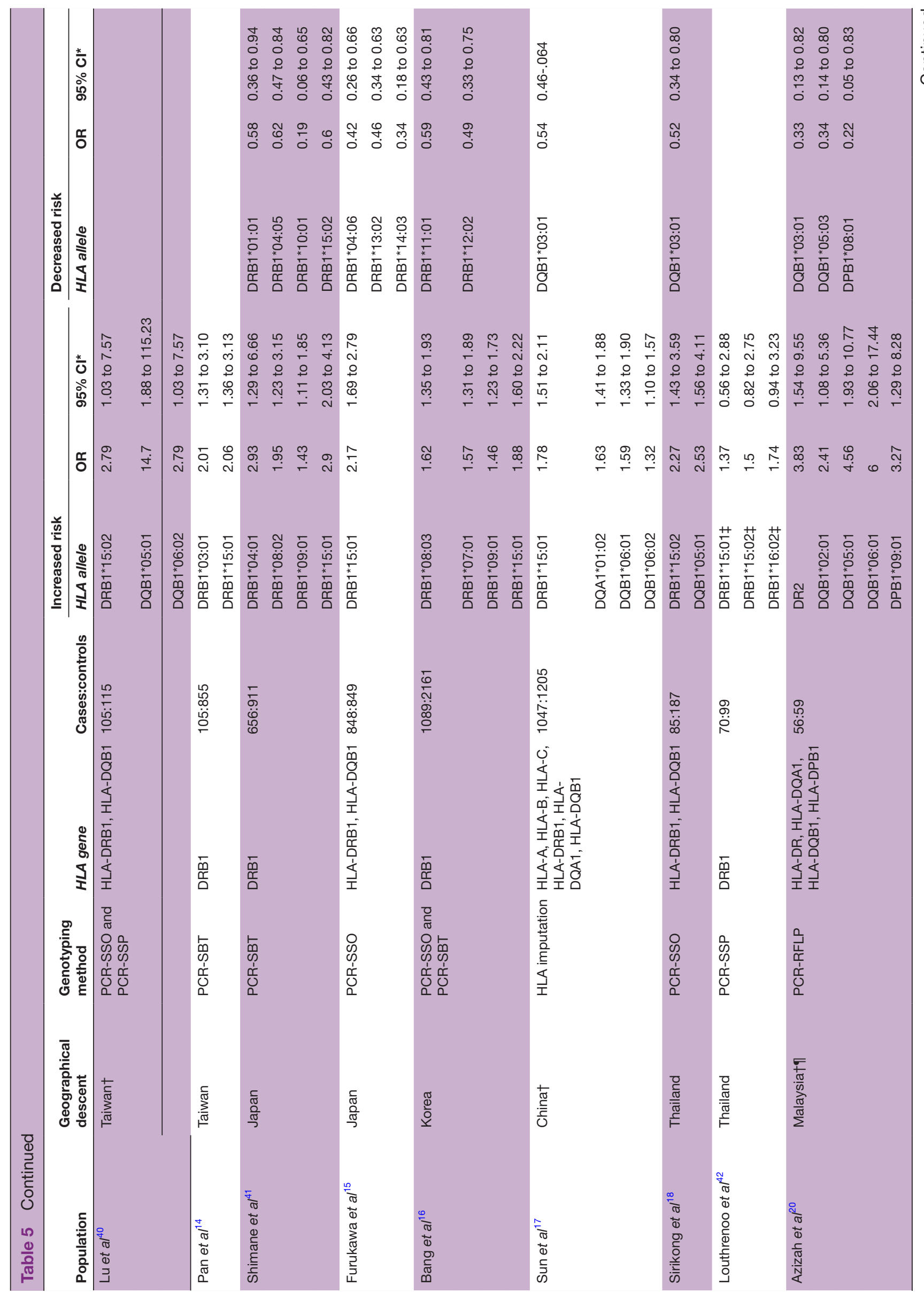

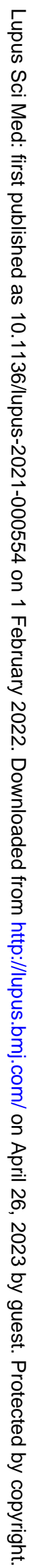




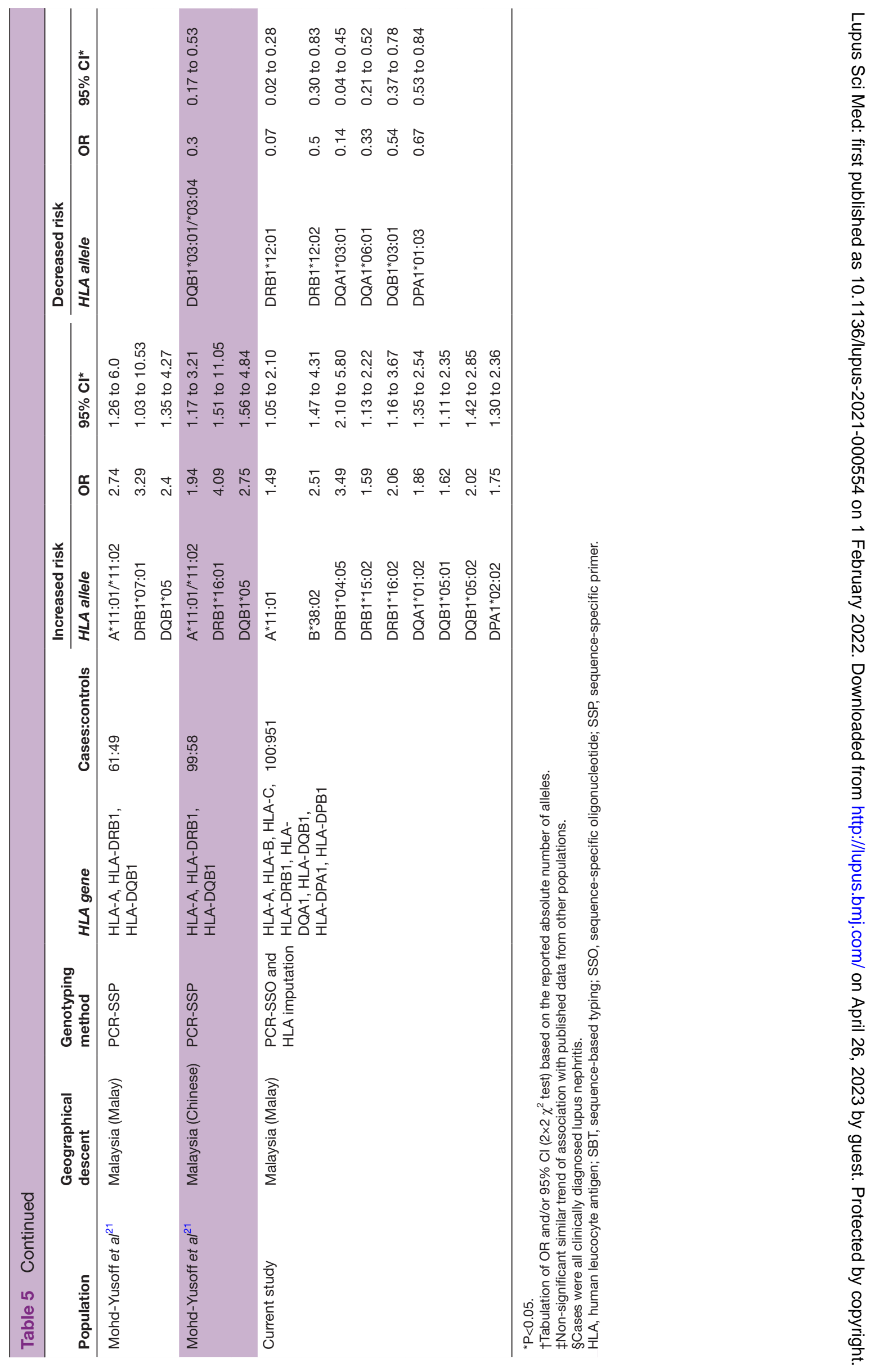


the 'autoimmune' haplotype 8.1 (HLA-A1/B8/Cw*07/ DRB1*03:01/DQB1*02:01) that has shown to be associated with SLE particularly among the Caucasians. ${ }^{46}$ However, the HLA-B*08 allele was rarely found in the Malaysian Malay patients with SLE (1\%) and the Malay general population.

HLA class II alleles have been repeatedly shown to display strong associations with SLE across the major histocompatibility complex region, particularly in the DRB1 and DQB1 loci. In the current study, we observed that HLA-DRB $1 * 15$ and DQB $1 * 03$ alleles were the most important susceptibility and protective variants for SLE in Malays and other ethnic groups, ${ }^{9} 11$ 13-18 20 29-31 34-38 40-43 suggesting that the genetic effects of these alleles are generalised to multiple ethnic populations of Caucasian, African and Asian descent.

It is worthy to mention that DRB $1 * 15$ polymorphisms were robustly associated with risk of SLE worldwide. However, further scrutinisation analysis at molecular level revealed a considerable heterogeneity of association signals between our population and those of Caucasian or East Asian origins. For instance, DRB1*15 risk factor seemed to be shared, but the risk signal often appeared to be population specific, that is, DRB1*15:01 in the Caucasians, Africans and East Asians. ${ }^{15-1729}{ }^{37}$ In contrast to DRB1*15:01, we observed a significant association with DRB1*15:02 in Southeast Asians and in Malaysian Malay SLE populations. ${ }^{1842}$ The differential role of these two DRB $1 * 15$ allele subtypes had not been clearly defined as DRB1*15:02 is a rare variant for Caucasian populations. On the other hand, the majority of previous studies in Asian populations investigated these alleles together as DR2 (or DR*15) serotypes. ${ }^{20}{ }^{43}$ Previous report on the amino acid sequence showed that these alleles differed at position 86 (a valine for DRB1*15:01 and glycine for DRB1*15:02). This amino acid constitutes the P1 pocket of the DR $\beta$ molecule and is considered to be crucial for antigen presentation. ${ }^{47}$

As shown in table 5, HLA-DRB1*03/*03:01 polymorphism and SLE was significant in patients with Caucasian and African origins, however, this susceptibility association was almost completely absent in the Asian studies except one report from Taiwan. ${ }^{14}$ Taking together our report with others, the genetic heterogeneity is common among SLE populations worldwide, especially between Caucasians, Africans and Asians. The identification of HLA genetic heterogeneity at molecular level (eg, classical four-digit) may thus enhance our understanding of mechanisms that lead to SLE pathogenesis in certain populations and allow for more precise and personalised diagnosis, prognosis and treatment stratification for the patients with SLE.

The genetic heterogeneity evident in patients with SLE of various ethnic populations may be attributed to the inheritance of different ancestral haplotypes that impact the development of SLE. ${ }^{48}$ In this study, the haplotype analysis revealed higher effect sizes in comparison to our univariate HLA allele's analysis and with relatively large
95\% CIs. For example, the commonly reported SLEassociated HLA-DRB1*15:02 DQB1*03:01 haplotype was significantly associated with risk of developing SLE in Malay patients with OR of 50 and $95 \%$ CI between 10.9 and 229.9. It is noteworthy that combination of multiple risk signals from different HLA loci observed in this study (eg, $A^{*} 11: 01 \sim \mathrm{DRB} 1 * 15: 02 \sim \mathrm{DQB} 1 * 05: 01, \mathrm{OR}=3.18,95 \%$ $\mathrm{CI}=1.53$ to 6.58$)$, did not demonstrate higher genetic effect, thus indicating low evidence for additive genegene interaction in Malay patients with SLE in our study.

HLA imputation is an approach of inferring an individual's HLA genotype using information about that individual's SNP genotype at sites flanking the classical HLA loci. This imputation allows us to immediately leverage massive SNP datasets which are readily available, with virtually no cost involved. Imputation also allows the transformation of SNP-based association into the HLA allele-level association, which may be biologically more informative. Furthermore, researchers can perform imputation to the level of protein/amino acid sequence within the HLA molecule, and predict the specific amino acid present, thus allowing for functional interpretations to be pursued.

In this study, we used the data from a large, wellcharacterised, population-based and ethnically matched normal controls for risk association testing with the SLE patient's cohort. ${ }^{23}{ }^{26}$ These normal individuals with Malay ethnicity were previously subjected to dense SNP genotyping using the Immunochip array ( 196 000 SNP markers) ${ }^{26}$ We then performed HLA imputation for classical HLA-DQA1, HLA-DPA1 and HLA-DPB1 genes for allelic association analysis. In our analysis, we convincingly validated DQA1*01:02 as a potential universal susceptibility risk factor for SLE development, consistent with the findings from Hungarian, Mexican, Han Chinese, Tunisian and Indian populations. ${ }^{17} 29343738$ In addition, we also newly identified DPA $1 * 02: 02$ as a susceptibility allele, while DPA $1 * 01: 03$ was identified as a protective allele with modest risk magnitudes in the Malay patients with SLE. An important limitation of our existing HLA-DQA1, HLADPA1 and HLA-DPB1 imputation, and of many previous studies, is that the present study was limited by lack of experimental HLA-DQA1, HLA-DPA1 and HLA-DPB1 genotype data for comparison and validation of concordance rates/imputation accuracy. Therefore, we tested our significant results with two published HLA-genotyped Malay control populations. ${ }^{44}{ }^{45}$ Our results showed that similar trend of associations for HLA-DPB1 gene using the Singapore Riau Malays, ${ }^{45}$ but were not significant after Bonferroni correction (online supplemental table S3). Similar association trends were also observed using the Southeast Asia Malay data from the Singapore Genome Variation Project. ${ }^{44}$ While we have convincingly replicated the findings for associated DPA $1 * 01: 03(\mathrm{OR}=0.52)$ and $\mathrm{DPA} 1 * 02: 02 \quad(\mathrm{OR}=1.60)$, we observed an opposite direction of risk effect for DQA $1 * 01: 02 \quad(\mathrm{OR}=1.85)$ using our normal controls versus the Southeast Asia Malays $(\mathrm{OR}=0.21$ ) (online supplemental table S3). We were unable to validate the results for DQA1*03:01 and 
DQA1*06:01 alleles as these alleles were not detected in the Southeast Asia Malays. ${ }^{44}$ As yet, we are not aware of large dataset with SNP and high-resolution HLA types in Southeast Asian populations, and argue that resources should be made available to generate large populationspecific reference panel to enable good quality HLA imputations.

SLE is a chronic autoimmune disease, characterised by multisystem organ inflammation, that is, skin, joints, kidneys, blood cells and nervous system. In this study, we used a large and well-defined patients set, for example, homogenous Malay ethnicity, all female patients with SLE with renal involvement (ie, presence of proteinuria $>0.5$ $\mathrm{g} /$ day or having active urinary sediments with $>5$ red cells per high-power field and/or casts, and in which $30 \%$ of these patients were having renal biopsy-proven lupus nephritis), and appropriate controls (ie, populationbased Malay individuals without history of autoimmune diseases/underlying chronic inflammatory diseases). On the other hand, owing to the systemic nature, and clinical phenotypic heterogeneity of the disease as well as overlapped abnormal laboratory assessment of renal profile such as presence of proteinuria and elevated creatinine levels, our study was not able to pinpoint the identified risk variants to be solely associated with involvement of renal manifestations in the patients with SLE. However, when we stratified the patients with SLE with or without lupus nephritis and tested the identified HLA class I and class II risk variants, we did not observe substantial differences for the ORs between these subgroups (data not shown) except for HLA-DRB1*04:05 (ie, SLE with lupus nephritis, OR=7.15, 95\% CI=3.60 to 14.22 vs SLE without lupus nephritis, $\mathrm{OR}=1.49 ; 95 \% \mathrm{CI}=0.69$ to 3.18 ) (online supplemental table S4). Furthermore, we did not investigate the environmental exposures and lifestyle factors that could determine susceptibility and/or progression of SLE, which could potentially create opportunities for primary and/or secondary prevention strategies.

This study demonstrates associations between broad arrays of classical HLA class I and class II genetic polymorphisms and SLE in the Malaysian female population. Using the data from this study, together with updated literature and their significant findings, we further support the notion that several HLA class II alleles (ie, DRB1*15, DQB1*03 and DQA1*01:02 alleles) are universally associated with risk of developing SLE in different ethnic populations and that these genetic effects are generalised to multiple ethnic populations of Caucasian, African and Asian descents. Further genetic and pharmacogenetics research in SLE development is crucial for understanding the pathogenetic mechanism involved which may pave a path for developing new and personalised therapeutic drugs for SLE.

\section{Author affiliations}

${ }^{1}$ Faculty of Pharmaceutical Sciences, No 1, Jalan Gading, UCSI Heights, Taman Connaught, UCSI University, Cheras, Kuala Lumpur, Malaysia
${ }^{2}$ Department of Medical Microbiology, Faculty of Medicine and Health Sciences, Universiti Putra Malaysia, Serdang, Selangor, Malaysia

${ }^{3}$ Immunogenetic Unit, Allergy and Immunology Research Centre, Ministry of Health Malaysia, National Institutes of Health Complex, Institute for Medical Research, Shah Alam, Selangor, Malaysia

${ }^{4}$ Department of Medicine, Division of Rheumatology, Karolinska Institutet and Karolinska University Hospital, Stockholm, Sweden

${ }^{5}$ Transplantation Immunology Unit, Allergy and Immunology Research Centre, Ministry of Health Malaysia, National Institutes of Health Complex, Institute for Medical Research, Kuala Lumpur, Malaysia

${ }^{6}$ Department of Medicine, Faculty of Medicine and Health Sciences, Universiti Putra Malaysia, Serdang, Selangor, Malaysia

${ }^{7}$ Allergy and Immunology Research Centre, Ministry of Health. National Institutes of Health Complex, Institute for Medical Research, Shah Alam, Selangor, Malaysia

Acknowledgements The authors would like to express our gratitude to Director General of Health Malaysia for supporting the work described in this article. The authors would like to thank the staffs of Nephrology Unit, Hospital Serdang and Immunology Unit, Department of Pathology, Faculty of Medicine and Health Sciences, Universiti Putra Malaysia for their dedication and excellent assistance throughout the study. We truly value the patients for their great participation. The authors would like to thank Dr Nor Asiah Muhamad and Ms Normi Mustapha for their assistance in logistic regression analysis, and Dr Nurul Aain Ahmad Fauzi for her assistance in reviewing all the analysed data. The authors would also like to thank Associate Professor Dr Leonid Padyukov for helpful discussion of the study results.

Contributors CLT acts as the guarantor of this article and take full responsibility for the work and/or the conduct of the study, has access to the data and controlled the decision to publish. MS: conceptualisation, methodology, data curation and investigation of patients with SLE, formal analysis, original draft preparation. CLT: conceptualisation, software, methodology, data curation and investigation of patients with SLE and normal controls, formal analysis, original draft preparation, project administration, funding acquisition and resources for the MyEIRA study. LKT: software, formal analysis. BTK: data curation, formal analysis. MA: supervision. AMdS: supervision. MA: conceptualisation, supervision, resources. SAN: conceptualisation, supervision, funding acquisition, resources. All authors reviewed and approved the final version prior to submission.

Funding This cohort was supported by funding from the Ministry of Higher Education, Malaysia, FRGS/2/2013/SKK01/UPM/02/8, with the Grant No. 5524400. The MyEIRA study was financially supported by the Ministry of Health, Malaysia (JPP-IMR 05-007, NMRR-07-299-572, NMRR-08-020-1975 and NMRR-11707-8010) and The Swedish National Research Council (DNR-348-2009-6468).

Competing interests None declared.

Patient consent for publication Not applicable.

Ethics approval Ethical approval was obtained from the Medical Research and Ethics Committee of the Faculty of Medicine and Health Sciences, Universiti Putra Malaysia (UPM) and Medical Research and Ethics Committee Ministry of Health Malaysia (Project ID: NMRR-14-1756-23234). All participants gave their informed written consent.

Provenance and peer review Not commissioned; externally peer reviewed. Data availability statement All data relevant to the study are included in the article or uploaded as supplementary information.

Supplemental material This content has been supplied by the author(s). It has not been vetted by BMJ Publishing Group Limited (BMJ) and may not have been peer-reviewed. Any opinions or recommendations discussed are solely those of the author(s) and are not endorsed by BMJ. BMJ disclaims all liability and responsibility arising from any reliance placed on the content. Where the content includes any translated material, BMJ does not warrant the accuracy and reliability of the translations (including but not limited to local regulations, clinical guidelines, terminology, drug names and drug dosages), and is not responsible for any error and/or omissions arising from translation and adaptation or otherwise.

Open access This is an open access article distributed in accordance with the Creative Commons Attribution Non Commercial (CC BY-NC 4.0) license, which permits others to distribute, remix, adapt, build upon this work non-commercially, and license their derivative works on different terms, provided the original work is properly cited, appropriate credit is given, any changes made indicated, and the use is non-commercial. See: http://creativecommons.org/licenses/by-nc/4.0/. 
ORCID iD

Chun Lai Too http://orcid.org/0000-0002-4079-8454

\section{REFERENCES}

1 Choi MY, Flood K, Bernatsky S, et al. A review on SLE and malignancy. Best Pract Res Clin Rheumatol 2017;31:373-96.

2 Justiz Vaillant AA, Goyal A, Bansal P. Systemic lupus erythematosus. Treasure Island, FL: StatPearls, 2021.

3 Gergianaki I, Bortoluzzi A, Bertsias G. Update on the epidemiology, risk factors, and disease outcomes of systemic lupus erythematosus. Best Pract Res Clin Rheumatol 2018;32:188-205.

4 Pons-Estel GJ, Ugarte-Gil MF, Alarcón GS. Epidemiology of systemic lupus erythematosus. Expert Rev Clin Immunol 2017;13:799-814.

5 Wang F, Wang CL, Tan CT, et al. Systemic lupus erythematosus in Malaysia: a study of 539 patients and comparison of prevalence and disease expression in different racial and gender groups. Lupus 1997;6:248-53

6 Kuo C-F, Grainge MJ, Valdes AM, et al. Familial aggregation of systemic lupus erythematosus and coaggregation of autoimmune diseases in affected families. JAMA Intern Med 2015;175:1518-26.

7 Gough SCL, Simmonds MJ. The HLA region and autoimmune disease: associations and mechanisms of action. Curr Genomics 2007:8:453-65.

8 Ghodke-Puranik Y, Niewold TB. Immunogenetics of systemic lupus erythematosus: a comprehensive review. J Autoimmun 2015;64:125-36.

9 Marchini M, Antonioli R, Lleò A, et al. Hla class II antigens associated with lupus nephritis in Italian SLE patients. Hum Immunol 2003;64:462-8.

10 McHugh NJ, Owen P, Cox B, et al. Mhc class II, tumour necrosis factor alpha, and lymphotoxin alpha gene haplotype associations with serological subsets of systemic lupus erythematosus. Ann Rheum Dis 2006;65:488-94.

11 Lundström E, Gustafsson JT, Jönsen A, et al. HLA-DRB1*04/*13 alleles are associated with vascular disease and antiphospholipid antibodies in systemic lupus erythematosus. Ann Rheum Dis 2013;72:1018-25.

12 Bettencourt A, Carvalho C, Leal B, et al. The Protective Role of HLA-DRB1(*)13 in Autoimmune Diseases. J Immunol Res 2015;2015:948723.

13 Hachicha H, Kammoun A, Mahfoudh N, et al. Human leukocyte antigens-DRB1*03 is associated with systemic lupus erythematosus and anti-SSB production in South Tunisia. Int $\mathrm{J}$ Health $\mathrm{Sci}$ 2018;12:21-7.

14 Pan CF, Wu CJ, Chen HH, et al. Molecular analysis of HLA-DRB1 allelic associations with systemic lupus Erythematous and lupus nephritis in Taiwan. Lupus 2009;18:698-704.

15 Furukawa H, Kawasaki A, Oka S, et al. Human leukocyte antigens and systemic lupus erythematosus: a protective role for the HLADR6 alleles DRB1*13:02 and *14:03. PLoS One 2014;9:e87792.

16 Bang S-Y, Choi J-Y, Park S, et al. Brief report: influence of HLA-DRB1 susceptibility alleles on the clinical subphenotypes of systemic lupus erythematosus in Koreans. Arthritis Rheumatol 2016;68:1190-6.

17 Sun J, Yang C, Fei W, et al. HLA-DQ $\beta 1$ amino acid position 87 and $\mathrm{DQB} 1{ }^{*} 0301$ are associated with Chinese Han SLE. Mol Genet Genomic Med 2018;6:541-6.

18 Sirikong M, Tsuchiya N, Chandanayingyong D, et al. Association of HLA-DRB1*1502-DQB1*0501 haplotype with susceptibility to systemic lupus erythematosus in Thais. Tissue Antigens 2002;59:113-7.

19 Kong NC, Nasruruddin BA, Murad S, et al. Hla antigens in Malay patients with systemic lupus erythematosus. Lupus 1994;3:393-5.

20 Azizah MR, Ainol SS, Kong NC, et al. Hla antigens in Malay patients with systemic lupus erythematosus: association with clinical and autoantibody expression. Korean J Intern Med 2001;16:123-31.

21 Mohd-Yusuf Y, Phipps ME, Chow SK, et al. HLA-A ${ }^{*} 11$ and novel associations in Malays and Chinese with systemic lupus erythematosus. Immunol Lett 2011;139:68-72.

22 Selvaraja M, Chin VK, Abdullah M, et al. HLA-DRB1“04 as a Risk Allele to Systemic Lupus Erythematosus and Lupus Nephritis in the Malay Population of Malaysia. Front Med 2020;7:598665.

23 Tan L-K, Mohd-Farid B, Salsabil S, et al. Hla-A, -B, -C, -DRB1 and -DQB1 alleles and haplotypes in 951 Southeast Asia Malays from Peninsular Malaysia. Hum Immunol 2016;77:818-9.

24 Hochberg MC. Updating the American College of rheumatology revised criteria for the classification of systemic lupus erythematosus. Arthritis Rheum 1997;40:1725.
25 Chun-Lai T, Padyukov L, Dhaliwal JS, et al. Shared epitope alleles remain a risk factor for anti-citrullinated proteins antibody (ACPA)-positive rheumatoid arthritis in three Asian ethnic groups. PLoS One 2011;6:e21069.

26 Tan LK, Too CL, Diaz-Gallo LM, et al. The spectrum of association in $H L A$ region with rheumatoid arthritis in a diverse Asian population: evidence from the MyEIRA case-control study. Arthritis Res Ther 2021;23:46

27 Jia X, Han B, Onengut-Gumuscu S, et al. Imputing amino acid polymorphisms in human leukocyte antigens. PLoS One 2013;8:e64683

28 Excoffier L, Lischer HEL. Arlequin suite VER 3.5: a new series of programs to perform population genetics analyses under Linux and windows. Mol Ecol Resour 2010;10:564-7.

29 Endreffy E, Kovács A, Kovács L, et al. Hla class II allele polymorphism in Hungarian patients with systemic lupus erythematosus. Ann Rheum Dis 2003;62:1017-8.

30 Ramal LM, López-Nevot MA, Sabio JM, et al. Systemic lupus erythematosus in southern Spain: a comparative clinical and genetic study between Caucasian and gypsy patients. Lupus 2004;13:934-40.

31 Armstrong DL, Zidovetzki R, Alarcón-Riquelme ME, et al. Gwas identifies novel SLE susceptibility genes and explains the association of the HLA region. Genes Immun 2014;15:347-54.

32 Flåm ST, Gunnarsson R, Garen T, et al. The HLA profiles of mixed connective tissue disease differ distinctly from the profiles of clinically related connective tissue diseases. Rheumatology 2015;54:528-35.

33 Vargas-Alarcón G, Salgado N, Granados J, et al. Class II allele and haplotype frequencies in Mexican systemic lupus erythematosus patients: the relevance of considering homologous chromosomes in determining susceptibility. Hum Immunol 2001;62:814-20.

34 Cortes LM, Baltazar LM, Lopez-Cardona MG, et al. Hla class II haplotypes in Mexican systemic lupus erythematosus patients. Hum Immunol 2004;65:1469-76.

35 de Holanda MI, Klumb E, Imada A, et al. The prevalence of HLA alleles in a lupus nephritis population. Transpl Immunol 2018;47:37-43.

36 Bhallil O, Ibrahimi A, Ouadghiri S, et al. Hla class II with lupus nephritis in Moroccan patients. Immunol Invest 2017;46:1-9.

37 Ayed K, Gorgi Y, Ayed-Jendoubi S, et al. The involvement of HLA -DRB1*, DQA1*, DQB1* and complement C4A loci in diagnosing systemic lupus erythematosus among Tunisians. Ann Saudi Med 2004;24:31-5.

38 Dedhia L, Pradhan V, Ghosh K, et al. Association of human leucocyte antigen (HLA) class II with systemic lupus erythematosis (SLE) patients from Western India. Meta Gene 2018;16:230-3.

39 Katkam SK, Rajasekhar L, Kutala VK. The influence of functional polymorphic positions of HLA-DR $\beta 1$ molecules on risk for South Indian systemic lupus erythematosus patients. Lupus 2018;27:991-1000

40 Lu LY, Ding WZ, Fici D, et al. Molecular analysis of major histocompatibility complex allelic associations with systemic lupus erythematosus in Taiwan. Arthritis Rheum 1997;40:1138-45.

41 Shimane K, Kochi Y, Suzuki A, et al. An association analysis of HLA-DRB1 with systemic lupus erythematosus and rheumatoid arthritis in a Japanese population: effects of ${ }^{*} 09: 01$ allele on disease phenotypes. Rheumatology 2013;52:1172-82.

42 Louthrenoo W, Kasitanon N, Wichainun R, et al. The genetic contribution of HLA-DRB5*01:01 to systemic lupus erythematosus in Thailand. Int J Immunogenet 2013;40:126-30.

43 Al-Motwee S, Jawdat D, Jehani GS, et al. Association of HLADRB $1{ }^{*} 15$ and HLADQB $1{ }^{*} 06$ with SLE in Saudis. Ann Saudi Med 2013;33:229-34.

44 Pillai NE, Okada Y, Saw W-Y, et al. Predicting HLA alleles from highresolution SNP data in three Southeast Asian populations. Hum Mol Genet 2014:23:4443-51.

45 Mark SJ, Erlich HA. 13th International Histocompatibility Workshop Anthropology/Human Genetic Diversity Joint Report - Chapter 3: Population Reports: Population Chinese, Malay and Thai from Singapore. In: Hansen J, ed. Immonobiology of the human MHC. proceedings of the 13th international histocompatibility workshop and conference. Seattle: IHWG Press, 2006: 36-7.

46 Goldberg MA, Arnett FC, Bias WB, et al. Histocompatibility antigens in systemic lupus erythematosus. Arthritis Rheum 1976;19:129-32.

47 Jones EY, Fugger L, Strominger JL, et al. Mhc class II proteins and disease: a structural perspective. Nat Rev Immunol 2006;6:271-82.

48 Hanscombe KB, Morris DL, Noble JA, et al. Genetic fine mapping of systemic lupus erythematosus MHC associations in Europeans and African Americans. Hum Mol Genet 2018;27:3813-24. 